\title{
VULNERABILIDAD GLOBAL ANTE EL CAMBIO CLIMÁTICO EN COMUNIDADES DE LA CUENCA LAGUNA DE PERLAS
}

Sheira Nanety Thomas Henríquez ${ }^{[1]}$ William Watlers ${ }^{[2]}$

\section{Resumen}

Se evaluó la vulnerabilidad global ante el cambio climático y se definieron líneas de acción de adaptación y mitigación a corto, mediano y largo plazo en once comunidades indígenas y afrodescendientes del municipio Laguna de Perlas, RAAS, Nicaragua. Por medio de la caracterización socioeconómica y biofísica de las once comunidades, validación y análisis de variables e indicadores de vulnerabilidad global ante el cambio climático, identificándose el estado de ocho ángulos de vulnerabilidad global en cada comunidad. La validación y análisis de variables demuestra una percepción comunal de los líderes de las once comunidades y alcance institucional que requiere un cambio de paradigma para enfrentar el cambio climático, revelando además que las once comunidades presentaron una vulnerabilidad global media (47.1\%), que sitúa a todos los tipos de vulnerabilidad en esta condición a excepción de la vulnerabilidad ideológico-cultural. Las comunidades con mayores vulnerabilidades son: La Fe (56.9\%), Raití Pura (50.3\%) y San Vicente (49.9\%). Se proponen algunas acciones de adaptación y mitigación a corto, mediano y largo plazo, las cuales requerirán la participación comunitaria e institucional a nivel municipal, regional y nacional para reducir las vulnerabilidades ante el cambio climático.

Palabras claves: vulnerabilidad, líneas de acción, adaptación, mitigación.

\section{Summary}

We assessed the global vulnerability to climate change and defined lines of action for adaptation and mitigation in the short, medium and long term in eleven Indigenous and Afrodescendant communities of the Pearl Lagoon municipality, RAAS, Nicaragua. Through the socioeconomic and biophysics characterization of the eleven communities, validation and analysis of variables and indicators of global vulnerability to climate change, it was possible to identify the status of eight angles of global vulnerability in each community. The validation and analysis of variables

\footnotetext{
${ }^{[1]}$ Máster en Desarrollo con Identidad. Coordinadora de Cooperación Externa, URACCAN recinto Bluefields. sheirathomas@gmail.com

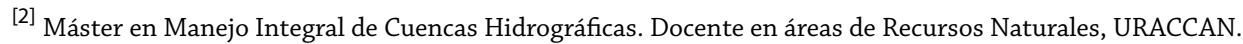


shows a community perception of the leaders of the eleven communities and the institutional reach that requires a change of paradigm to deal with the climate change; it also reveals that the eleven communities had a median global vulnerability (47.1\%), which places all types of vulnerability in this condition, excepting the ideological and cultural vulnerability. The communities with higher vulnerabilities are: $\mathrm{La} \mathrm{Fe}(56.9 \%)$, Raiti Pura (50.3\%) and San Vicente (49.9\%). Some actions are proposed for adaptation and mitigation in the short, medium and long term, which will require community and institutional participation at the municipal, regional and national levels to be able to reduce vulnerabilities to climate change.

Keywords: vulnerability, lines of action, adaptation, mitigation.

\section{Introducción}

Este artículo retoma el alcance del desarrollo humano para el siglo XXI, supeditado al fenómeno del cambio climático. Cambios que afectarán por la interacción entre riesgo y vulnerabilidad global de una comunidad o sociedad basada en la perspectiva de Wilches-Chaux (1993); quien estima que las sociedades pertenecientes a los países en desarrollo y los ciudadanos más pobres, son los que en situaciones de riesgos producto del cambio climático, serán los más vulnerables (PNUD, 2007).

Desde esta perspectiva la vulnerabilidad de comunidades del tercer mundo no se centra en la carencia de recursos económicos y materiales, sino cómo esta privación menoscaba la importancia de la prevención de riesgos. Es así, que las medidas preventivas deben articularse para contribuir con el buen viviri[3] de la población en un período corto o mediano (5 - 10 años), según sus necesidades sentidas.

En este contexto se considera que: 1) Los estudios realizadas en el territorio no han permitido conocer ecuánimemente el estado de los ángulos de vulnerabilidades en las comunidades $y$, por consiguiente las acciones actuales difieren de las soluciones integrales que se requieren para abordar el problema de la vulnerabilidad; 2) Persiste la deforestación, pérdida de la biodiversidad, erosión de los suelos y la contaminación hídrica, así como otros usos del terreno que afectan directamente las principales actividades de subsistencia: la pesca, agricultura y caza; 3 ) El nivel de pobreza y alta dependencia de los bienes y servicios que proporcionan los ecosistemas, mayormente presionados por el ser humano y los efectos del cambio climático.

Por consiguiente, el estudio propone acciones de mitigaciones, adaptación a corto, mediano y largo plazo con la finalidad de reducir la vulnerabilidad y mejorar la relación beneficio/costo de las medidas planteadas.

[3] Es la vida en armonía con la naturaleza y los seres espirituales protectores. Es buscar la convivencia en comunidad, donde todos los integrantes se preocupan por todos. Donde lo más importante no es lo material, ni el dinero, sino la vida (del Cid, 2010). 


\section{Revisión de literatura}

El cambio climático es una importante variación estadística que persiste durante un largo período de tiempo, según el Intergovernmental Panel on Climate Change (IPCC). $\mathrm{Si}$ bien los pueblos indigenas inciden en mayor proporción a este cambio, comparten con el resto del mundo los impactos de estas variaciones sobre los ecosistemas, Cunningham et al. (2009).

Según Jiménez, citado por Watler (2008), la vulnerabilidad se define como el proceso para determinar los componentes críticos, débiles o suceptibles de daño o interrupciones de eedificaciones instalaciones y las medidas de emergencia y mitigación a tomarse ante una amenaza específica o un grupo de ellas. Para este estudio, se utilizó la concepción de vulnerabilidad global planteada por Wilches-Chaux en compilación realizada por Maskrey (1993); el cual hace una división en: natural, física, económica, social, política, técnica, cultural, educativa, ecólogica, e institucional. Asimismo, el informe de la alcaldía municipal de Laguna de Perlas y Beer G. y Vanegas S. en el diagnóstico en doce comunidades indígenas y afrodescendientes.

El IPCC (2007) define la adaptación al cambio climático como: "Iniciativas y medidas encaminadas a reducir la vulnerabilidad de los sistemas naturales y humanos ante los efectos reales o esperados de un cambio climático."

Maskrey (1993) por su parte, conceptualiza la mitigación como:

medidas de intervención dirigidas a reducir o atenuar el riesgo. La mitigación es el resultado de la decisión a nivel político de un nivel de riesgo aceptable obtenido de un análisis extensivo del mismo y bajo el criterio de que dicho riesgo es imposible reducirlo totalmente.

En síntesis, el análisis de vulnerabilidad es una herramienta que permite conocer y sensibilizar sobre los riesgos que enfrentan las comunidades ante los cambios climáticos y el rol de las entidades que están involucradas en las tareas de gestión de riesgo.

\section{Materiales y métodos}

En el estudio se aplicó una metodología mixta con enfasis en el paradigma sociocrítico. La investigación se desarrolló en el año 2010 en once comunidades del municipio Laguna de perlas, RAAS, como son: Haulover, Kahkabila, Marshall Point, Pearl Lagoon, Orinoco, La Fe, Raití Pura, Brown Bank, Set Net Point, Awas y San Vicente. 
La población y la muestra se definieron en base al número de viviendas que hay en cada comunidad. Siendo la población el número total de viviendas y la muestra fue definida haciendo uso del muestreo aleatorio estratificado.

Cuadro No. 1.

\begin{tabular}{|c|c|c|}
\hline Comunidad & Número de vivienda & Muestra/estrato de once comunidades \\
\hline Awas & 15 & 3 \\
\hline Brown Bank & 32 & 7 \\
\hline Haulover & 214 & 48 \\
\hline Kahkabila & 75 & 17 \\
\hline La Fe & 24 & 5 \\
\hline Marshall Point & 38 & 9 \\
\hline Orinoco & 126 & 28 \\
\hline Raití Pura & 66 & 15 \\
\hline Set Net Point & 38 & 9 \\
\hline San Vicente & 13 & 3 \\
\hline Pearl Lagoon & 496 & 112 \\
\hline Total & $\mathbf{1 1 3 7}$ & $\mathbf{2 5 6}$ \\
\hline
\end{tabular}

En el proceso de validación participaron funcionarios del INAFOR, MAGFOR, MINED, URACCAN, Defensa Civil e INPESCA.

Las encuestas fueron aplicadas a hombres y mujeres mayores de edad en las once comunidades. Los grupos focales se realizaron con los líderes comunitarios de las once comunidades. Las entrevistas se aplicaron a representantes de las instituciones locales: Ministerio de Educación, Centro de salud, Wildlife Conservation Society y Ayuda en Acción.

Se utilizó fuentes secundarias respaldadas con resultados de información primaria para realizar la caracterización socioeconómica y biofísica, e información primaria para describir la percepción comunitaria y el alcance de las instituciones gubernamentales y no gubernamentales locales. Para el análisis de vulnerabilidad, la vulnerabilidad global se dividió en vulnerabilidad física (VF); económica (VEN); social (VS); política-institucional (VP-I); técnica (VT); ideológico-cultural (VI-C); educativa (VED) y ecológica-eventos naturales (VE-EN). Para cada ángulo de vulnerabilidad se definió variables e indicadores considerando las características particulares de la zona de estudio. Los indicadores fueron validados y caracterizados retomando la propuesta de Watler (2008), haciendo uso de una escala de ponderación lineal de o a 4, es decir muy baja o nula (o), baja (1), media (2), alta (3), muy alta (4). Se parte de la premisa que entre mayor es la valoración asignada mayor es el grado de vulnerabilidad para esa variable. 
De la misma manera se consideró la escala de valoración de la vulnerabilidad (Cuadro No.2) expresada en forma porcentual por Jiménez (2007).

Cuadro No. 2. Caracterización y valoración de la vulnerabilidad en porcentaje.

\begin{tabular}{|c|c|}
\hline Porcentaje de vulnerabilidad & Caracterización de la vulnerabilidad \\
\hline $0.00-19.99$ & Muy baja \\
\hline $20.00-39.99$ & Baja \\
\hline $40.00-59.99$ & Media \\
\hline $60.00-79.99$ & Alta \\
\hline $80.00-100$ & Muy alta \\
\hline
\end{tabular}

Además, se definió durante la validación de indicadores el peso relativo para cada tipo de vulnerabilidad. El cuadro No. 3 muestra los pesos relativos de cada tipo de vulnerabilidad.

Cuadro No.3. Peso relativo por cada tipo de vulnerabilidad.

\begin{tabular}{|c|c|}
\hline Vulnerabilidad (xi) & Peso relativo (wi) \\
\hline Física (VF) & 0.11 \\
\hline Económica (VEN) & 0.13 \\
\hline Social (VS) & 0.14 \\
\hline Política-Institucional (VP-I) & 0.12 \\
\hline Técnica (VT) & 0.09 \\
\hline Ideológica-cultural (VI-C) & 0.15 \\
\hline Educativa (VED) & 0.16 \\
\hline Ecológica-eventos naturales (VE-EN) & 0.12 \\
\hline $\boldsymbol{\Sigma}$ Total & 1.00 \\
\hline
\end{tabular}

Una vez validada la propuesta de variables e indicadores y definida la ponderación del peso relativo para cada tipo de vulnerabilidad, se procedió a establecer los parámetros o guías de campo para cada indicador. Así como los instrumentos metodológicos de cinco entrevistas semi-estructuradas, aplicadas a funcionarios de las instituciones gubernamentales y no gubernamentales; once grupos focales dirigidos a los líderes comunitarios (un grupo focal por comunidad) y 256 encuestas definidas a través del muestreo aleatorio estratificado.

Obtenida la información de la valoración de los indicadores se determinó la puntuación de cada indicador por comunidad. Estos valores fueron agrupados en un cuadro resumen que socializa información de cada tipo de vulnerabilidad, a través del cual se determinó el promedio de la vulnerabilidad de cada comunidad y su porcentaje de vulnerabilidad, para luego caracterizarlo. 
Una primera estimación porcentual de vulnerabilidad global ante el cambio climático por cada comunidad, sin ponderar se obtuvo utilizando la siguiente ecuación: $\mathrm{VG} \%=(\mathrm{VF} \%+\mathrm{VEN} \%+\mathrm{VS} \%+\mathrm{VP}-\mathrm{I} \%+\mathrm{VT} \%+\mathrm{VI}-\mathrm{C} \%+\mathrm{VED} \%+\mathrm{VE}-\mathrm{EN} \%) / 8$

Los valores obtenidos fueron comparados con la escala de valoración establecida para determinar el grado de vulnerabilidad global. Esta vulnerabilidad global fue estimada a través de la siguiente ecuación lineal ponderada:

$$
V G=\sum_{i=1}^{6}\left(x_{i} * w_{i}\right)
$$

Es decir VG $=\left(V^{*}{ }^{*} w i\right)+\left(V^{*}{ }^{*} w i\right)+(V S * w i)+(V P-I * w i)+(V T * w i)+(V I-C * w i)$ $+\left(\mathrm{VED}^{*}\right.$ wi $)+\left(\mathrm{VE}-\mathrm{EN}^{*}\right.$ wi $)$

Para una mejor representación de la información obtenida se plasmó a través de un gráfico los valores obtenidos de los indicadores de las vulnerabilidades por comunidad llamado línea base, la cual facilitó la selección de los indicadores con valoración mayor o igual a dos ( $\geq 2)$; considerados los indicadores prioritarios al momento de establecer las líneas de acción de adaptación y mitigación.

\section{Resultados y discusión}

\section{Aspectos socioeconómicos y biofísicos del municipio}

Basados en los datos oficiales del INIDE (2005), trece son las comunidades principales del municipio Laguna de Perlas, conformadas en siete comarcas por un total de 10,676 personas, distribuidas en 5,260 hombres y 5,316 mujeres.

La organización tradicional comunitaria de todas las etnias del municipio está constituida por los miembros prominentes y respetables de la comunidad, los cuales componen el concejo de ancianos, jueces, síndicos y whistas (INIFOM, S/f). Las actividades económicas que prevalecen son la pesca, agricultura y la caza, mencionadas de acuerdo a su orden de importancia.

En lo que respecta a salud, nueve son las unidades de salud del municipio de las cuales seis unidades atienden las once comunidades en estudio logrando una cobertura aproximada del $60 \%$ de la población y el restante $40 \%$ la reciben a través de brigadas médicas o medicina tradicional (Alcaldía municipal Laguna de Perlas, 2010).

En el municipio, hay cuarenta y cuatro centros educativos (nivel pre-escolar, primario y secundario) de los cuales quince están localizados dentro del territorio de las once comunidades. Hay una alta cobertura de Educación Preescolar y Primaria, y 
una baja cobertura de Educación Secundaria. La Educación Primaria y Secundaria del municipio se caracterizan por una baja retención y aprobación escolar (MINED, 2008).

Existe dos vías de transporte, el acuático y terrestre, siendo la vía acuática la más utilizada. Las estructuras tradicionales de las viviendas están siendo reemplazadas por concreto. En cuanto a los materiales de construcción, para el caso de los techos predomina el zinc con $94.72 \%$, en las paredes el material Plycem con $78.28 \%$, seguido de madera con $57.87 \%$, y predomina el piso de cemento con $12.58 \%$ (Beer y Vanegas, 2007).

Según el Instituto Nicaragüense de Fomento Municipal (INIFOM; S/f), el clima corresponde a la clasificación de selva tropical húmeda, con valores máximos de precipitación anual de $4000 \mathrm{~mm}$, temperaturas que no superan los $37^{\circ} \mathrm{C}$ y la más baja ha sido superior a los $18^{\circ} \mathrm{C}$. Los vientos alisios son los predominantes y casi persistentes en todo el año. La humedad relativa refleja una media anual de $89 \%$. Debido a la ubicación del municipio y sus características socio-naturales y culturales, exhiben amenazas y riesgos de huracanes, tormentas, inundaciones y lluvias constantes.

El municipio se ubica en la zona geomorfológica de la Planicie Costanera del Atlántico, la cual se caracteriza por un relieve dominantemente plano a fuertemente ondulado, con pendientes que fluctúan entre o-15\%. El uso del suelo del municipio se divide en bosque latifoliado cerrado, bosque latifoliado abierto, uso agropecuario, las tierras sujetas a inundación, vegetación arbustiva y mangle.

El municipio Laguna de Perlas se encuentra ubicado en la cuenca $\mathrm{N}^{\circ}{ }_{50}$ (cuenca de laguna de Perlas). Este territorio es un complejo lagunar de gran importancia biológica y económica de unos $550 \mathrm{~km} 2$ incluyendo la laguna de Top Lock $30 \mathrm{~km} 2$ aproximados). Su flora es típica de un bosque tropical húmedo, donde coexiste una biodiversidad. Se han encontrado más de 250 especies de flora, más de 255 especies de aves, 60 especies de mariposas y una cantidad desconocida de mamíferos y reptiles.

\section{Percepción comunitaria y alcance institucional}

Las prácticas de cooperación y solidaridad comunitaria se están perdiendo, llegando a percibirse en su generalidad como acciones organizativas de los líderes comunitarios o como acciones fortuitas ante eventos extremos u ocasiones especiales (muerte y desastres naturales). Resultado que corresponde con lo reportado por Cunningham et.al. (2010), quienes destacan que la solidaridad y la cohesión comunitarias han descendido entre los individuos y entre las comunidades en los últimos años, lo que modifica las relaciones entre los individuos, en la redistribución comunal de los recursos, pero también en las fortalezas para enfrentar en grupo los fenómenos naturales. 
Repite una líder comunitaria de Awas:

Antes se trabajaba de forma colaborativa, toda la comunidad trabajaba: los niños, jóvenes y adultos pero ahora no. Antes todos hacíamos limpieza comunitaria, ahora no". Un líder de Laguna de Perlas menciona "son pocas las prácticas de cooperación, pero se puede decir en su mayoría que cada quien está por su cuenta.

La funcionalidad de las organizaciones existentes en la comunidad (comités de riesgo, educación y salud) no es óptima a causa del poco o nulo apoyo de las instituciones gubernamentales. La causa radica en que sólo se conforman los comités, pero no se les capacita y si hay capacitación, todo termina en teoría sin la ejecución de acciones concretas, además de que la preparación y acción no es preventiva al evento sino cuando hay un desastre.

Si bien existen actitudes de búsqueda de un bien común después de un desastre o en vísperas de este en las comunidades estudiadas, es necesario rescatar algunas acciones colectivas debilitadas u olvidadas en la actualidad, de tal manera que el capital social comunitario al que refiere el informe del PNUD (2005) se fortalezca.

La percepción fatalista tiene opiniones encontradas, por un lado algunos consideran que los desastres naturales son "obra de Dios" y otros consideran que son obra del ser humano. Partiendo de esto, es notoria la necesidad de un trabajo conjunto (comunitarios e instituciones) para fortalecer la capacidad y alcance de las estructuras comunitarias. Además, la paulatina pérdida de prácticas de cooperación y la persistencia del fatalismo de lo divino, deben ser revertidas para reducir las vulnerabilidades.

El alcance institucional refleja que el tema de cambio climático no es un tema de abordaje directo: "Se toca de forma transversal". No se cuenta con personal capacitado directamente en el tema, el conocimiento que se posee se obtiene principalmente del autoestudio, existiendo muchos vacíos, por esta razón, no hay una designación presupuestaria, y en caso de la existencia, los fondos de cobertura son insuficientes para las necesidades existentes.

Este punto de análisis, refleja que se debe fortalecer el entendimiento institucional del tema de cambio climático, comprendiendo primeramente que el problema es complejo y requiere soluciones transdisciplinarias y esfuerzos interinstitucionales. Por lo tanto, es preciso que exista un reconocimiento del papel preponderante que juegan las instituciones locales y su papel de incidencia, sin obviar la necesidad de un mayor fortalecimiento de las debilidades que estas poseen. 


\section{Vulnerabilidad global de las once comunidades}

Cuadro No. 4. Vulnerabilidad global de las once comunidades indígenas y afrodescendientes ponderada.

\begin{tabular}{|c|c|c|c|c|c|c|c|c|c|c|c|}
\hline \multirow[b]{2}{*}{ Comunidad } & \multicolumn{11}{|c|}{ Vulnerabilidad ponderada } \\
\hline & 岇 & 忌 & $\simeq$ & $\overline{\grave{s}}$ & 5 & $\stackrel{\text { ب }}{\supset}$ & 모 & $\begin{array}{l}\text { 岕 } \\
\text { 岁 }\end{array}$ & $\begin{array}{l}\text { 음 } \\
\text { 틀 } \\
\text { 혼 }\end{array}$ & 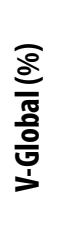 & 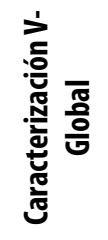 \\
\hline Awas & $\tilde{o}$ & $\hat{o}$ & ก̃ & ฮี & กี & $\stackrel{\circ}{\circ}$ & $\stackrel{n}{0}$ & กั & $\stackrel{\infty}{+}$ & $\stackrel{\infty}{\mathscr{q}}$ & Media \\
\hline Brown Bank & $\tilde{o}$ & ठே. & ก̃ & $\tilde{o}$ & กี & $\bar{\circ}$ & $\stackrel{m}{0}$ & $\check{\circ}$ & $\stackrel{9}{=}$ & 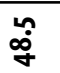 & Media \\
\hline Halouver & $\tilde{o}$ & $\tilde{o}$ & ๙̊. & $\tilde{o}$ & ชี & $\bar{\circ}$ & $\dddot{o}$ & กี & $\stackrel{\infty}{-}$ & 字 & Media \\
\hline Kahkabila & $\tilde{o}$ & 官 & ơ & ơ & ก̊ & $\bar{\sigma}$ & no & กั & $\stackrel{\circ}{i}$ & ชุ่ & Media \\
\hline La Fe & $\stackrel{+}{\circ}$ & : & on & $\tilde{o}$ & ก̃ & $\check{\circ}$ & சே & 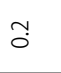 & $\stackrel{n}{i}$ & ํํें & Media \\
\hline Marshall Point & $\approx$ & $\stackrel{n}{0}$ & ฮี & $\tilde{o}$ & ๙̃ & $\because$ & $\approx$ & ๙ & $\stackrel{0}{\circ}$ & ळi & Ваја \\
\hline Orinoco & กี & $\tilde{o}$ & $\tilde{0}$ & กี & $\tilde{o}$ & $\bar{\circ}$ & $\tilde{o}$ & $\dddot{o}$ & $\stackrel{9}{\circ}$ & $\stackrel{9}{\dot{\gamma}}$ & Media \\
\hline Pearl Lagoon & $\check{\circ}$ & $\tilde{o}$ & $\tilde{o}$ & $\tilde{o}$ & õ & $\bar{\sigma}$ & $\tilde{o}$ & $\overline{0}$ & $\stackrel{\circ}{\circ}$ & กุ & Media \\
\hline Raití Pura & $\dddot{o}$ & 官 & กี & $\tilde{o}$ & $\tilde{o}$ & $\check{\circ}$ & சே & ชุ & $\stackrel{\circ}{i}$ & $\stackrel{m}{\circ}$ & Media \\
\hline San Vicente & ¿ே & $\dddot{o}$ & ๙̃ & $\tilde{o}$ & กี & $\overline{0}$ & ப் & กี & $\stackrel{\circ}{i}$ & के & Media \\
\hline Set Net Point & $\tilde{o}$ & $\tilde{o}$ & $\tilde{o}$ & 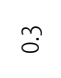 & กี & $\stackrel{\circ}{\circ}$ & $\tilde{o}$ & $\tilde{0}$ & $\stackrel{9}{-}$ & $\begin{array}{l}\stackrel{0}{0} \\
\stackrel{\infty}{+}\end{array}$ & Media \\
\hline Promedio & $\stackrel{\circ}{\stackrel{m}{m}}$ & $\stackrel{\bar{n}}{m}$ & $\underset{\sim}{\sim}$ & 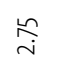 & $\stackrel{\infty}{i}$ & 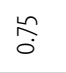 & 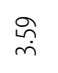 & $\stackrel{\infty}{i}$ & $\stackrel{\infty}{\circ}$ & $\overline{\dot{y}}$ & Media \\
\hline \multicolumn{12}{|c|}{$\begin{array}{l}\text { Donde VF= vulnerabilidad física, VEN= Vulnerabilidad económica, VS=Vulnerabilidad social, } \\
\text { VP-I=Vulnerabilidad político-institucional, VT=Vulnerabilidad técnica, VI-C=Vulnerabilidad } \\
\text { ideológico-cultural, VED= Vulnerabilidad educativa, VE-EN: Vulnerabilidad ecológica-eventos } \\
\text { naturales, M= Media y B= Baja }\end{array}$} \\
\hline
\end{tabular}


La estimación de vulnerabilidad global ha situado a todos los tipos de vulnerabilidades en una caracterización media a excepción del tipo ideológica-cultural, y esto se debió a la disponibilidad que manifiestan todas las comunidades en participar activamente en las acciones pre y post desastre, a pesar de ello, resalta el fatalismo de lo divino en más de la mitad de la población encuestada de las once comunidades (53\% de la población). Esto confirma el enunciado de Cunningham en cuanto a la presencia del "castigo de Dios", concepción que según Maskrey (1993), es transformada en incapacidad para enfrentar los problemas adecuadamente.

Se considera que la disposición de la población en participar en acciones pre y postdesastres permitirá con una buena organización, educación y recursos económicos, reducir la incapacidad que puede atribuir esta idea fatalista, sin evadir la necesidad de definir acciones concretas que reduzcan o eliminen esta percepción. Condicionando entonces, la fortaleza de esta percepción en las debilidades sociales, educativas, técnicas, ecológicas, culturales y económicas; estableciendo así la interrelación existente entre los ángulos de vulnerabilidad y por consiguiente la importancia de la división de la vulnerabilidad global que plantea Wilches-Chaux para un mejor entendimiento de las causas de los problemas, y la definición de actividades que contrarresten todas las vulnerabilidades identificadas para generar una mejor capacidad de respuesta ante riesgos.

La actual vulnerabilidad de las comunidades son: a) Su dependencia de los recursos naturales para su subsistencia, generalmente con poca diversificación técnica; b) Localización inadecuada de asentamientos humanos (localizadas a menos de $200 \mathrm{~m}$ de la costa), se suman los materiales de construcción inadecuados en sus viviendas ante cualquier evento natural; c) El bajo nivel de ingreso económico, poca diversificación de las actividades productivas y falta de valor agregado a los productos ofertados, inexistencia de una cadena de comercialización que incentive el comercio justo, el limitado acceso a incentivos económicos e insumos para la conservación ambiental y el acceso de las comunidades al pago por servicios ambientales; d) Falta de servicios básicos de salud y educación, y de prácticas de cooperación comunitaria; e) Escasa presencia de las instituciones y las que se encuentran poseen poca capacidades de respuesta ante un desastre, f) Falta de inversión en el proceso educativo, para la generación de conocimientos que reduzcan los riesgos e incertidumbres; y g) Poco dominio de técnicas y tecnologías que aporten a la prevención y reducción de riesgos, tomando en cuenta el conocimiento tradicional de los pueblos para pronosticar y mitigar los efectos del cambio climático.

Ante esto, se ve la necesidad de fortalecer el liderazgo comunitario, la salud preventiva occidental y tradicional, la autonomía comunitaria, capacidad organizacional, economía comunitaria, la revitalización y promoción del conocimiento ancestral; asimismo, se requiere reorientar características culturales de riesgo (regular la urbanización dentro de la franja de los $200 \mathrm{~m}$, materiales de construcción de viviendas), 
priorizar gestiones hacia la diversificación de los sectores económicos productivos con políticas y programas dirigidos al incremento de la económica familiar a mediano plazo; impulsar una educación ambiental sostenible y de gestión de riesgo, el incremento tecnológico, acceso a medios de comunicación, mayor presencia, incidencia y capacidad institucional y finalmente una intervención conjunta (comunidades y las instituciones gubernamentales y no gubernamentales), para juntos tomar medidas de adaptación y mitigación a los efectos del cambio climático.

Partiendo de este escenario se procedió a la definición de las líneas de acción que retoman la necesidad de transformar o mejorar la calidad de vida de la población y reducir sus vulnerabilidades, todo en conjunto con las instituciones locales gubernamentales y no gubernamentales, y los comunitarios para el logro de la misma.

\section{Propuestas de línea de acción en la adaptación y mitigación}

Las propuestas de líneas de acción de adaptación y mitigación se elaboraron a partir de los resultados del análisis de la vulnerabilidad global de las once comunidades indígenas y afrodescendientes del municipio Laguna de Perlas, de la estrategia nacional ambiental del cambio climático realizado por el Gobierno de Nicaragua (2010) y las líneas propuestas por Cunningham et al (2010). Partiendo de esto, las líneas establecidas parten de los siguientes objetivos:

\section{Objetivos de las líneas}

Se constituyen los siguientes objetivos para la implementación de las líneas de acción:

- Implementar un programa de educación comunitaria e institucional continua sobre el tema de cambio climático.

- Rescatar las prácticas ancestrales sostenibles socialmente, ambiental y culturalmente.

- Promover la gestión institucional y comunitaria para la búsqueda de soluciones ante los posibles impactos del cambio climático.

- Promover medidas de adaptación en la población de las once comunidades del municipio Laguna de Perlas.

Las líneas definidas en el Cuadro No. 4, corresponden a períodos de corto (5 años), mediano (10 años) y largo plazo (15 años); las cuales se plantean tomando en cuenta la flexibilidad y capacidad de adecuarse a la dinámica y realidad de las once comunidades indígenas y afrodescendientes del municipio. Líneas de intervenciones que deben ejecutarse de forma paulatina y que deben ir acompañadas de procesos de sistematización y evaluaciones continúas, con el propósito de mantener una permanente reflexión y ajuste de actividades en caso de que requiera una redefinición de la planificación, según resultados obtenidos. 
Cuadro No. 5. Propuestas de líneas de acción de adaptación y mitigación de las once comunidades indígenas y afrodescendientes del municipio Laguna de Perlas.

\begin{tabular}{|c|c|c|c|c|c|c|c|c|c|c|c|c|c|c|c|}
\hline \multirow{3}{*}{$\begin{array}{l}\text { Líneas de acción de } \\
\text { adaptación y mitigación }\end{array}$} & \multicolumn{15}{|c|}{$\begin{array}{l}\text { Tiempo para aplicar las líneas de acción } \\
\text { adaptación y mitigación al cambio climático }\end{array}$} \\
\hline & \multicolumn{5}{|c|}{5 años } & \multicolumn{5}{|c|}{10 años } & \multicolumn{5}{|c|}{15 años } \\
\hline & 1 & 2 & 3 & 4 & 5 & 6 & 7 & 8 & 9 & 10 & 11 & 12 & 13 & 14 & 15 \\
\hline \multicolumn{16}{|c|}{$\begin{array}{l}\text { Objetivo No. 1. Implementar un programa de educación comunitaria e institucional } \\
\text { continua sobre el tema de cambio climático }\end{array}$} \\
\hline \multicolumn{16}{|l|}{$\begin{array}{l}\text { Realizar capacitaciones de cambio climáti- } \\
\text { co con un enfoque integral: conservación } \\
\text { ambiental, gestión de riesgo, prevención y } \\
\text { mitigación ante el cambio climático, salud, } \\
\text { educación y otros. }\end{array}$} \\
\hline \multicolumn{16}{|l|}{$\begin{array}{l}\text { Diseñar y difundir programas radiales } \\
\text { orientados a la conservación ambiental, } \\
\text { modelos y materiales de construcción de } \\
\text { vivienda, cambio climático y vulnerabili- } \\
\text { dades locales, medidas de adaptación al } \\
\text { cambio climático y gestión de riesgo. }\end{array}$} \\
\hline \multicolumn{16}{|l|}{$\begin{array}{l}\text { Capacitar a comunitarios y líderes reli- } \\
\text { giosos en el tema de cambio climático: } \\
\text { causas, acciones preventivas, acciones } \\
\text { post desastre. }\end{array}$} \\
\hline \multicolumn{16}{|l|}{$\begin{array}{l}\text { Capacitar a comunitarios productores en } \\
\text { sistemas agroforestales, acuícola y agricul- } \\
\text { tura ecológica para la diversificación de } \\
\text { aplicación de técnicas. }\end{array}$} \\
\hline \multicolumn{16}{|l|}{$\begin{array}{l}\text { Desarrollar un plan de resiliencia que } \\
\text { aborde los aspectos culturales, espirituales } \\
\text { y productivos. }\end{array}$} \\
\hline \multicolumn{16}{|l|}{$\begin{array}{l}\text { Identificar y capacitar a capacitadores de } \\
\text { las áreas temáticas integrales del cambio } \\
\text { climático de salud, educación, medio } \\
\text { ambiente y desarrollo comunitario. }\end{array}$} \\
\hline \multicolumn{16}{|c|}{$\begin{array}{l}\text { Objetivo No. 2. Rescatar las prácticas ancestrales sostenibles socialmente, } \\
\text { ambiental y cultural }\end{array}$} \\
\hline \multicolumn{16}{|l|}{$\begin{array}{l}\text { Sistematizar y promover la revitalización } \\
\text { de las prácticas de cooperación comunita- } \\
\text { ria tradicionales. }\end{array}$} \\
\hline \multicolumn{16}{|l|}{$\begin{array}{l}\text { Sistematizar y divulgar las prácticas y técni- } \\
\text { cas ambientales ancestrales. }\end{array}$} \\
\hline $\begin{array}{l}\text { Realizar investigaciones científicas sobre } \\
\text { conocimientos tradicionales de los pue- } \\
\text { blos indígenas y afrodescendientes sobre: } \\
\text { prácticas agrícolas, manejo del clima, suelo } \\
\text { y agua, adaptación y mitigación local } \\
\text { ante el cambio climático, salud, relaciones } \\
\text { naturaleza-humanos, entre otros. }\end{array}$ & & & & & & & & & & & & & & & \\
\hline
\end{tabular}




\begin{tabular}{|c|c|c|c|c|c|c|c|c|c|c|c|c|c|c|c|}
\hline \multirow{3}{*}{$\begin{array}{l}\text { Líneas de acción de } \\
\text { adaptación y mitigación }\end{array}$} & \multicolumn{15}{|c|}{$\begin{array}{c}\text { Tiempo para aplicar las líneas de acción adaptación } \\
\text { y mitigación al cambio climático }\end{array}$} \\
\hline & \multicolumn{5}{|c|}{5 años } & \multicolumn{5}{|c|}{10 años } & \multicolumn{5}{|c|}{15 años } \\
\hline & 1 & 2 & 3 & 4 & 5 & 6 & 7 & 8 & 9 & 10 & 11 & 12 & 13 & 14 & 15 \\
\hline \multicolumn{16}{|c|}{$\begin{array}{l}\text { Objetivo No. 3. Promover la gestión institucional y comunitaria para la búsqueda } \\
\text { de soluciones ante los posibles impactos del cambio climático. }\end{array}$} \\
\hline \multicolumn{16}{|l|}{$\begin{array}{l}\text { Fortalecer las capacidades de organiza- } \\
\text { ción y gestión de financiamiento dentro } \\
\text { de las instituciones y líderes comunales. }\end{array}$} \\
\hline \multicolumn{16}{|l|}{$\begin{array}{l}\text { Gestionar la adquisición y transferencia } \\
\text { de técnicas y tecnologías de monitoreo, } \\
\text { seguimiento y evacuación (pre y post } \\
\text { eventos extremos). }\end{array}$} \\
\hline \multicolumn{16}{|l|}{$\begin{array}{l}\text { Gestionar la construcción y manteni- } \\
\text { miento de albergues comunitarios y } \\
\text { centro de salud. }\end{array}$} \\
\hline \multicolumn{16}{|l|}{$\begin{array}{l}\text { Promover redes de negocio de los } \\
\text { rubros aprovechados culturalmente } \\
\text { dentro de la comunidad. }\end{array}$} \\
\hline \multicolumn{16}{|l|}{$\begin{array}{l}\text { Gestionar financiamiento para la elabo- } \\
\text { ración y aplicación de planes de gestión } \\
\text { de riesgo dentro de las comunidades. }\end{array}$} \\
\hline \multicolumn{16}{|l|}{$\begin{array}{l}\text { Fortalecer la capacidad empresarial } \\
\text { comunitaria. }\end{array}$} \\
\hline \multicolumn{16}{|c|}{$\begin{array}{l}\text { Objetivo No. 4. Promover medidas de adaptación y de mitigación en la población } \\
\text { de las once comunidades del municipio Laguna de Perlas }\end{array}$} \\
\hline \multicolumn{16}{|l|}{$\begin{array}{l}\text { Regular la urbanización de viviendas } \\
\text { dentro de los } 200 \text { m de la zona costera } \\
\text { dentro de las comunidades a través de } \\
\text { una normativa municipal. }\end{array}$} \\
\hline \multicolumn{16}{|l|}{$\begin{array}{l}\text { Promover el modelo de salud regional, } \\
\text { en el marco de la articulación de la } \\
\text { medicina tradicional y occidental. }\end{array}$} \\
\hline \multicolumn{16}{|l|}{$\begin{array}{l}\text { Conformar y fortalecer los comités } \\
\text { comunitarios de gestión de riesgos. }\end{array}$} \\
\hline $\begin{array}{l}\text { Proporcionar mayor acceso a incenti- } \\
\text { vos económicos familiares e insumos } \\
\text { que promueva el desarrollo ambiental, } \\
\text { económico, y social. }\end{array}$ & & & & & & & & & & & & & & & \\
\hline
\end{tabular}




\section{Conclusiones}

En referencia a la caracterización socioeconómica y biofísica de las once comunidades:

La información biofísica y socioeconómica permite la identificación directa de causas de vulnerabilidades ante el cambio climático en las once comunidades.

En referencia al análisis de vulnerabilidad global:

La percepción comunitaria y el alcance institucional denotan la existencia del fatalismo, pérdida de prácticas de cooperación y solidaridad, la poca funcionalidad de instituciones locales y el imperante concepto del cambio de clima (acciones de mitigación y adaptación), por lo tanto, las soluciones a estas debilidades radica en las decisiones y acciones antropogénicas de formación e incidencia.

La comunidad La Fe, seguido de las comunidades Raiti Pura y San Vicente son las más vulnerables.

La vulnerabilidad de las comunidades identifican las causas a razón de siete de los ocho ángulos de vulnerabilidad global (VF, VEN, VS, VP-I, VT, VED y VE-EN), indicando la necesidad del desarrollo de acciones a nivel local; pero, no empezando desde cero, sino construyendo sobre las ya existentes y probadas a ese nivel. Acciones que aborden el orden: ecosistemas, culturales, base económica, geografía, clima y gestión de riesgo.

En lo concerniente a proponer líneas de acción de mitigación y adaptación ante el cambio climático:

Las líneas de acción deberían ser ejecutadas retomando la complejidad de la realidad comunitaria, la adaptabilidad, la continua reflexión y aprendizaje, y sobre todo el involucramiento de todos los actores.

Las líneas de acción propuestas en la investigación presentan acciones graduales. Estas propuestas reflejan la necesidad de impulsar un enfoque sectorial (agrícola, hídrico, forestal, social, económico) en las comunidades, con acciones que coadyuven a empatar el desarrollo social y la sustentabilidad ambiental.

\section{Lista de referencia}

Alcaldía Municipal. Informe de campo Laguna de Perlas (no publicado). Nicaragua. p 7-50. 
Beer G., Vanegas S. (2007). Diagnóstico para la demarcación de las doce comunidades indígenas y afrodescendientes de la cuenca de Pearl Lagoon. Informe de campo. Bluefields, Nicaragua. p 17- 64 .

Cunningham, M., Mairena D., \& Pacheco, M. (2010). Cambo climático: medidas de adaptación en comunidades de las Regiones Autónomas de la Costa Caribe de Nicaragua. Editorial Arista. Managua, Nicaragua. p 8-24.

Cunningham, M., Mairena D., \& Mairena, E. (2009). Guía sobre cambio climático y pueblos indígenas: un cuaderno de referencias. Managua, Nicaragua. p 17-18.

Del Cid, Víctor Manuel (2010). Glosario de ciencias sociales y pueblos indígenas. Managua, Nicaragua. $232 \mathrm{p}$.

Gobierno de Nicaragua. (2010). Estrategia nacional ambiental y del cambio climático, plan de acción 2010-2015. $27 \mathrm{p}$.

Instituto Nicaragüense de Fomento Municipal (INIFOM). (s/f). Ficha municipal Laguna de Perlas. En línea http://www.inifom.gob.ni/municipios/documentos/ ATLANTICO\%2OSUR/laguna_de_perlas

Instituto Nacional de Información de Desarrollo. (2005). Laguna de perlas en cifras. En línea: http://www.inide.gob.ni/censos2005/CifrasMun/RAAS/Laguna\%20 de\%2oPerlas.pdf

Maskrey Andrew (1993). Los desastres no son naturales. En línea: http://www.desenredando.org/public/libros/1993/ldnsn/LosDesastresNoSonNaturales-1.o.o

MINED (2008). Índice del estado educativo municipal IEEM. En línea: http://www.mined. gob.ni/PDFo8/ieem2007_actualizadomayoo8_a.pdf

Programa de las Naciones Unidas para el Desarrollo (PNUD; 2005) Informe de Desarrollo Humano 2005: Las Regiones Autónomas de la Costa Caribe: ¿Nicaragua asume su diversidad? Managua, Nicaragua.

Watler, W. (2008). Análisis de vulnerabilidad a la contaminación del recurso hídrico en la subcuenca del río Siquirres, Costa Rica. Turrialba, CATIE, Tesis Mag. Sc. 212 p.

Wilches-Chaux, G. (1993). La vulnerabilidad global. In Maskrey, A. (comp.). Los desastres no son naturales. Bogotá, Colombia, La Red. p 9-50. 